To appear in the Proceedings of the ICFA-HB2004'04 Workshop

Bensheim, Germany, Oct. 18-22, 2004

\title{
Adjusted Field Profile for the Chromaticity Cancellation in FFAG Accelerators"
}

\author{
Alessandro G. Ruggiero \\ Brooldwowen National Laborator; Collider-Accelerator Department, \\ PO Box 5000, Upton, Long Island, New York 11973, USA
}

\begin{abstract}
Abstrict. In an earlier report [1] we have reviewed four major rules to design the lattice of Fixed-Field AltematingGradient (FFAG) accelerators. One of these rules deals with the search of the Adjusted Field Profile, that is the field non-linear distribution along the length and the width of the accelerator magnets, to compensate for the chromatic behavior, and thus to reduce considerably the variation of betatron tunes during acceleration over a large momentum range. The present report defines the method for the search of the Adjusted Field Profile.
\end{abstract}

Reywords: FFAG Accelerators, Magnet Field, Beam Dynamics, Proton Beams, Chromaticity.

PACS: $29.20 \cdots \mathrm{C}$

\section{INTRODUCTION}

The FFAG ring is made of a number of identical periods shown in Fig. 1. According to one of the rules [1]. each period is made of a FDF triplet. There is a reference trajectory that according to another nule [1] corresponds to the injection momentum value $\mathrm{p}_{0}$. The reference trajectory is made of arcs of circle in the scctor magnets $\mathrm{F}$ and $\mathrm{D}$ with constant curvature, and of straight lines in the drift regions $S$ and $g$. The opposing exit and entrance planes of the neighboring sector magnets are parallel to each other, thus, the entrance and exit angles of the reference trajectory with each magnet are identically zero. Nevertheless

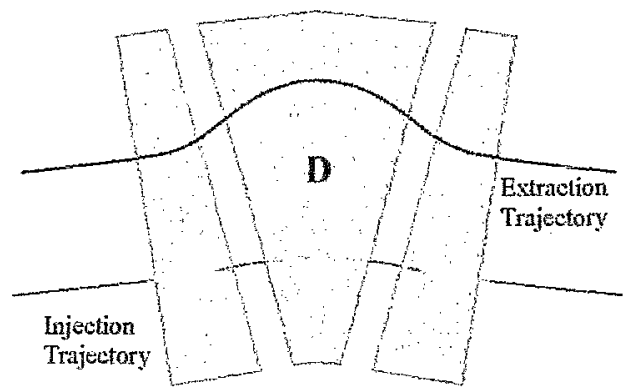

FIGURE 1. The FFAG FDF Period with Inject. (Reterence) and Extract. Orbits trajectories of other momentum values in a NonScaling Lattice like the one discnssed here, have a more complicate behavior and are not arcs of circle since they cross regions with different bending field. Moreover these trajectories always leave or enter magnets at non-vanishing angles.

\section{EQUATIONS OF MOTION}

We adopt a curvilinear coordinate system $(x, s, y)$ where $s$ is the path length along the reference (injection) trajectory, and $\mathrm{x}, \mathrm{y}$ are respectively the horizontal and vertical displacements from the reference trajectory.

The equations of motion for any particle with momentum $p$ and electric charge $q$ are derived from the (exact) Hamiltonian

$$
\begin{aligned}
H= & -q A_{s} / c-(1+h x)\left[p^{2}-\left(p_{x}-q A_{x} / c\right)^{2}\right. \\
& \left.-\left(p_{y}-q A_{y} / c\right)^{2}\right]^{12}
\end{aligned}
$$

where $c$ is the light velocity, $h=h(s)$ the curvature of the reference trajectory, and $p_{\mathrm{x}} \mathrm{p}_{\mathrm{y}}$ are the transverse components of the particle momentum canonically conjugated to $x, y$.

The equations of motion are

\footnotetext{
"Work performed nnder the auspices of the U.S. Department of Energy
} 
$\begin{array}{ll}\mathrm{p}_{\mathrm{x}}^{\prime}=-\partial H / \partial \mathrm{x} & \mathrm{x}^{\prime}=\partial \mathrm{H} / \partial \mathrm{p}_{\mathrm{x}} \\ \mathrm{p}_{\mathrm{y}}^{\prime}=-\partial \mathrm{H} / \partial \mathrm{v} & \mathrm{y}^{\prime}=\partial \mathrm{H} / \partial \mathrm{p}_{\mathrm{y}}\end{array}$

with a prime denoting differentiation with respect to $s$ $\left({ }^{\prime} \equiv d / d s\right)$. Expanding the square root at the $r$. h. side of Eq. (1) and retaining only up to quadratic order terms give:

$$
\begin{aligned}
H= & -q A_{S} / c-(1+h x) p+ \\
& +\left(p_{x}-q A_{x} / c\right)^{2} / 2 p+ \\
& +\left(p_{y}-q A_{y} / c\right)^{2} / 2 p
\end{aligned}
$$

after having also dropped higher order terms.

We assume that the magnetic ficld distribution in the proximity of the reference trajectory is given solely by the longitudinal component $A_{3}$ of the vector potential, whereas identically $A_{x}=A_{y}=0$. In this case the equations of motion become

$$
\begin{aligned}
& x^{\prime \prime}=(q / p c) \partial A_{s} / \partial x+h \\
& y^{\prime \prime}=(q / p c) \partial A_{s} / \partial y
\end{aligned}
$$

This is correct only as long $A_{s}$ does not vary with the longitudinal position within the length of one magnet: otherwise a solenoid field component is introduced that should be taken into account.

\section{MAGNETIC FIELD COMPONENTS}

With the chosen curvilinear coordinate system, the components $B_{x}$ and $B_{y}$ of the magnetic field are

$$
\begin{aligned}
& (1+h x) B_{y}=\quad \partial A_{s} / \partial x \\
& (1+h x) B_{x}=-\partial A_{s} / \partial y
\end{aligned}
$$

and the equations of motion are now

$x^{\prime \prime}=(q / p c) B_{y}(1+h x)+h$
$y^{\prime \prime}=-(q / p c) B_{x}(1+h x)$

Formally, using complex notation, we can write

$B=B_{y}+i B_{x}$

$z=x+i y$

Quite generally

$B(x)=B_{0}+G(z) z$

where $B_{0}$ is the bending field on the reference trajectory, and $\mathrm{G}(\mathrm{z})$. We have

$$
B_{\mathrm{x}}=\operatorname{Imaginary}\{B(\mathrm{z})\}
$$$$
\mathrm{B}_{\mathrm{y}}=\operatorname{Real}\{\mathrm{B}(\mathrm{z})\}
$$

\section{CHROMATIC EFFECTS ON THE HORIZONTAL PLANE}

Let us consider now the motion on the mid-plane where $y=0$,

$\mathrm{x}^{*}=(\mathrm{g} / \mathrm{pc})\left(\mathrm{B}_{0}+\mathrm{Gx}\right)(1+\mathrm{hx})+\mathrm{h}$

But the bending condition on the reference orbit is

$\left(\mathrm{q} \mathrm{B}_{0} / \mathrm{p}_{0} \mathrm{c}\right)=-\mathrm{h}$

Introduce also the relative momentum deviation $p=$ $p_{0}(1+\delta)$ with $\delta=0$ for the reference (injection) orbit. Neglecting the higher order term $(\mathrm{q} / \mathrm{pc}) \mathrm{G} \mathrm{h} \mathrm{x}^{2}$, the horizontal equation of motion (11) reduces to

$$
\begin{aligned}
x^{\prime \prime}= & (q / p c)\left(h B_{0}+G\right) x+h+q B_{0} / p c \\
= & -h^{2}\left(1+G / h B_{0}\right) x /(1+\delta)+ \\
& +h \delta /(1+\delta)
\end{aligned}
$$

or, introducing the field index $\mathrm{n}=\mathrm{G} / \mathrm{h} \mathrm{B}_{0}$

$x^{\prime \prime}+h^{2}(1+n) x /(1+b)=h \delta /(1+\delta)$

Like $G$, also the freld index $n$ is in general a function of $\mathrm{x}$ (and $\mathrm{y}$ ). Similarly, in proximity of the mid-plane, the vertical equation of motion is

$$
y^{2}-h^{2} n y /(1+0)=0
$$

Equations (14, 15) can be in general solved numerically. In the special case the field index $n$ is constant, the solution can be found analytically, and the well-known mafrix method can be used to estimate the usual lattice functions. Because of the explicit momentum dependence at the denominator of both terms, the solution exhibits strong dispersive and chromatic effects.

\section{FORMULATION OF THE PROBLEM}

Let us consider the most general case where the field index is a nonlinear function of both $x$ and $s$, namely $n=n(x, s)$. We know that, at any location $s$, for each momentum value $\delta$ there is one and only one unique solution $\mathrm{x}=\mathrm{x}(\mathrm{o}, \mathrm{s})$, and by imversion, topologically, 8 can be taken as a function of $x$ and $s$, namely $\delta=\delta(x, s)$. We pose the following problem: Determine the freld distribution, namely $n=n(x, s)$. that compensates (mostly) exactly the momentum dependence of $(1+\delta)$ at the denominator. We shall write such solution as 
$n(x, s)=\quad n_{0}\lfloor l+\not \partial(x, s)]$

where $n_{0}$ is related to the gradient $G_{0}=n_{n} h B_{0}$ on the reference trajectory. If such field distribution is found then the Eq.s $(14,15)$ of motion reduce simply to

$\mathrm{x}^{\prime \prime}+\mathrm{h}^{2} \mathrm{x} /(1+\delta)+\mathrm{h}^{2} \mathrm{n}_{0} \mathrm{x}=\mathrm{h} \delta /(1+\delta)$

$y^{\prime \prime}-h^{2} n_{0} y$

$=0$

Notice that this approach does not cancel the momentum dependence of the curvature term, nor the constant dispersive term at the $r$. h. side.

\section{INTEGRATION OF THE EQUATION OF MOTION}

The solution of either Eq. (14) or Eq. (17a) can be written as the sum of two contributions

$\mathrm{x}(\delta, s)=\mathrm{x}_{0}(\delta, s)+\mathrm{u}(\delta, s)$

where $x_{c}$ is a particular solution of the inhomogeneous Eq. (14 or 17a) that has a periodic belevior, and $u$ is the free betatron solution that satisfies the associated homogenous equation. The solution of equation (17a) is straightorward and can be found with the wellknown matrix notation. After that once the closed orbit is found, the solution can be inverted either numerically or symbolically as done by MATHEMA TICA [2]. The imersion will provide the dependence of the momentum deviation with the radial displacement at any given locations,

$\delta=\delta(x, s)$

that we can insert back in the field expression to derive the equation of motion

$$
\begin{aligned}
x^{\prime \prime} & +h^{2}\left\{1+n_{0}[1+\delta(x, s)]\right\} x /(1+\delta)= \\
& =h \delta /(1+\delta)
\end{aligned}
$$

Proceeding backward, this corresponds to the field distribution on the mid-plane given by

$$
B(x, s)=B_{0}+G_{0}[1+\delta(x, s)] x
$$

\section{PROVE OF THE VALDITY OF THE PROPOSED APPROACH}

Equation (20) combined to (21), though nonlinear, can be integrated. The solution is indeed expected to have rcmoved the chromatic behavior causcd by the momentura dependence of the magnetic field. The integration of (20) can be attempted in two ways:

(i) Numerical integration by starting with some initial conditions $\mathrm{x}_{0}(0)$ and $\mathrm{x}_{0}{ }^{\prime}(0)$ and looking for $a$ solution that closes at the end of the period. To facilitate the search for the closed orbit solution, the initial conditions can be set from the determined solution $x_{6}(0, s)$ of the linear equation $(17 a)$.

(ii) By direct substitution of the originally determined solution $x_{4}(\delta, s)$ of the linear equation (17a) into equation (20), and by recording the relative deviation.

Either of these two methods, as we have successfully demonstrated with the use of MA THEMA TICA itself, will have proven the validity of the approach.

\section{CONCLUSIONS}

We have applied the method outlined in this report to calculate the Adjusted Field Profle for the cancellation of the chromatic behavior in FFAG accelerators adopting Non-Scaling Latfices. We have applied the method to three different projects: (1) a 1.5-GeV proton FFAG for Beam Power Upgrade of the Alternating Gradient Synchrotron at Brookhaven National laboratory $[41 ;(2)$ a $1-\mathrm{GeV} 10-\mathrm{MW}$ at Proton Driver in an unspecified site [5]; and (3) a $250-\mathrm{MeV}$ proton FFAG as a Medical Facility. We urge the reader to inspect also the other reports in the Reference list to acquire more details about the application and the results of the method described here.

\section{REFERENCES}

1. A.G. Ruggieto, "Design Criteria of a Proton $\mathrm{HEAG}$ Accelerator", Contribution to FFAG'04 Worlshop Proceedings, October 13-16, 2004, KKK, Tsukuba, Japan.

2. MATHEMATICA, version 5.0, Wolfram Research, http:/www.wolfram.com.

3. A.G. Ruggiero "Sharp Edge Effects of the Magnets of a FFAG Accelerator": Contribution to FFAG'04 Workshop Procesdings, October 13-16, 2004, KEK, 'Isukuba, Japan.

4. A.G. Ruggiero, "1.5-GeV FFAG Proton Accelerator for the AGS Upgrade". Invited lalk to EPAC-04, July 611, 2004, Lucerne, Switzerland.

5. A.G. Ruggiero, "A 1-GeV 10-MWatt Proton Driver" Invited rall to ICFA-HB2004, October 18-22, 2004, Bensheim, Germany. 


\section{DISCLAIMER}

This report was prepared as an account of work sponsored by an agency of the United States Government. Neither the United States Government nor any agency thereof, nor any of their employees, nor any of their contractors, subcontractors or their employees, makes any warranty, express or implied, or assumes any legal liability or responsibility for the accuracy, completeness, or any third party's use or the results of such use of any information, apparatus, product, or process disclosed, or represents that its use would not infringe privately owned rights. Reference herein to any specific commercial product, process, or service by trade name, trademark, manufacturer, or otherwise, does not necessarily constitute or imply its endorsement, recommendation, or favoring by the United States Government or any agency thereof or its contractors or subcontractors. The views and opinions of authors expressed herein do not necessarily state or reflect those of the United States Government or any agency thereof. 